\title{
O mindful eating modifica o comportamento alimentar em indivíduos com excesso de
}

\author{
peso? \\ Does mindful eating modify eating behavior in individuals with excess weight? \\ ¿La alimentación consciente cambia la conducta alimentaria en personas con sobrepeso?
}

Recebido: 02/03/2021 | Revisado: 07/03/2021 | Aceito: 10/03/2021 | Publicado: 18/03/2021

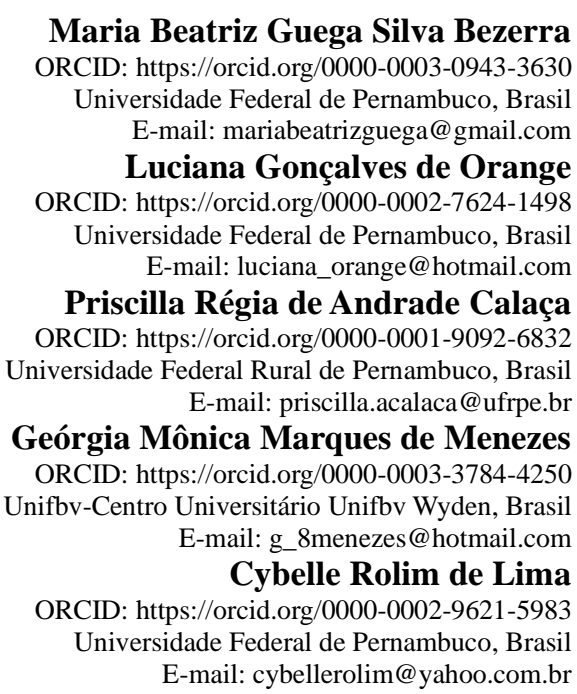

\begin{abstract}
Resumo
As estratégias tradicionais no controle ao excesso de peso, como a utilização de dietas restritivas e incentivo à prática de exercícios, à longo prazo, parecem não ser eficientes tendo em vista que muitas vezes não promovem mudanças de comportamento alimentar. Sendo assim, estratégias comportamentais, como o Mindful eating que é a habilidade de comer com atenção plena, sem julgamento ou crítica, auxiliando o indivíduo a ter uma melhor relação com a comida através da sintonia entre mente, corpo e alimentação, parece ser uma alternativa eficaz para consolidação de uma alimentação mais consciente. O objetivo deste estudo foi realizar uma revisão integrativa da literatura sobre os principais protocolos de Mindful eating utilizados em pesquisas com indivíduos com excesso de peso para mudança de comportamento alimentar. A pesquisa realizou-se nos principais bancos de dados: PubMed, ScienceDirect, TripDatabase e Scielo. Foram selecionados 8 artigos por preencherem os critérios de inclusão para a produção da revisão. Foi realizada a análise de diferentes protocolos de mindful eating, os quais demonstraram como principais benefícios, variando entre os estudos, a redução/manutenção do peso corporal; melhora no comportamento alimentar e diminuição nos episódios do transtorno de compulsão alimentar (TCA), aumento da atenção plena e da alimentação consciente. Dentre os protocolos avaliados, o MB-EAT foi o mais utilizado nas pesquisas com estes objetivos. Destaca-se a importância de mais pesquisas que utilizem esta estratégia e seus diferentes protocolos, ressaltando a análise dos efeitos que eles proporcionam no comportamento alimentar.
\end{abstract}

Palavras-chave: Obesidade; Ciências da nutrição; Atenção plena; Dieta; Protocolos.

\begin{abstract}
Traditional strategies for the control of excess weight, such as the use of restrictive diets and the encouragement of physical exercise, do not seem to be effective in the long term, as they often fail to promote a change in eating behavior. Therefore, behavioral strategies, such as mindful eating, which is being mindful of what one eats without judgment or criticism and helps an individual to have a better relationship with food through the establishment of harmony among mind, body and eating, seems to be an effective alternative for the consolidation of more conscious eating. The aim of the present study was to perform an integrative review of the literature on the main mindful eating protocols used in studies involving individuals with excess weight to achieve a change in eating behavior. Searches were performed in the PubMed, ScienceDirect, TRIP and Scielo databases. Eight articles met the inclusion criteria for the present review. The benefits of the different mindful eating protocols varied among the studies. The main benefits were a reduction in or maintenance of weight, an improvement in eating behavior, a reduction of episodes of binge eating and increases in mindfulness and conscious eating. MB-EAT was the most used protocol among the studies
\end{abstract}


included in the present review. Further studies involving different mindful eating strategies are needed for a better analysis of the effects of such protocols on eating behavior.

Keywords: Obesity; Nutritional Sciences; Mindfulness; Diet; Protocols.

\section{Resumen}

Las estrategias tradicionales para controlar el exceso de peso, como el uso de dietas restrictivas y el incentivo al ejercicio, a largo plazo, parecen no ser eficientes, dado que muchas veces no promueven cambios en la conducta alimentaria. Por tanto, las estrategias conductuales, como Mindful Eating, que es la capacidad de comer de forma consciente, sin juzgar ni criticar, ayudando al individuo a tener una mejor relación con la comida a través de la armonía entre mente, cuerpo y comida, parece ser una alternativa eficaz para Consolidar una dieta más consciente El objetivo de este estudio fue realizar una revisión integradora de la literatura sobre los principales protocolos de alimentación Mindful utilizados en investigaciones con personas con sobrepeso para cambiar la conducta alimentaria. La investigación se realizó en las principales bases de datos: PubMed, ScienceDirect, TripDatabase y Scielo. Se seleccionaron ocho artículos por cumplir con los criterios de inclusión para producir la revisión. Se realizó el análisis de diferentes protocolos de alimentación consciente, que demostraron como principales beneficios, variando entre estudios, la reducción / mantenimiento del peso corporal; mejora de la conducta alimentaria y disminución de los episodios de trastorno por atracón (BED), aumento de la atención plena y la alimentación consciente. Entre los protocolos evaluados, el MB-EAT fue el más utilizado en investigaciones con estos objetivos. Se destaca la importancia de seguir investigando utilizando esta estrategia y sus diferentes protocolos, destacando el análisis de los efectos que aportan sobre la conducta alimentaria.

Palabras clave: Obesidad; Ciencias de la Nutrición; Atención plena; Dieta; Protocolos.

\section{Introdução}

A mudança no padrão alimentar na população brasileira vem contribuindo para um crescente aumento das condições crônicas, isso ocorre devido ao cenário atual onde as pessoas estão submetidas à várias ocupações que demandam rapidez, menor esforço físico e menor atividade física, o que faz com que procurem alimentações mais rápidas e não tenham tempo de praticar exercícios físicos (Pinheiro, Freitas \& Corso, 2004). Destacando o aumento do consumo de carnes e alimentos industrializados, fast-foods, que possuem uma quantidade elevada de sal, ácidos graxos trans e gorduras saturadas, sendo grandes fatores de risco para as doenças crônicas não transmissíveis (DCNT) (Levy, Claro, Mondini, Sichieri \& Monteiro, 2012; World Health Organization, 2010).

As dietas restritivas para perda de peso, especialmente nos casos de excesso de peso, são padrões de alimentação que seguem recomendações nutricionais vigentes para atender as necessidades específicas, procurando promover o controle do peso, podendo estar intimamente ligadas ao atual conceito sociocultural de beleza, na maioria das vezes adotadas sem nenhum embasamento científico (Foxcroft, 2013). Como, por exemplo, aquelas que adotam medidas: pula refeições, altera a quantidade ingerida de alimentos, evita grupos alimentares considerados "engordativos", o que faz com que a alimentação seja vista como uma grande inimiga, provocando ansiedades, angústias e medos irracionais (Alvarenga, Polacow \& Scagliusi, 2016; Rozin Ashmore. \& Markwith, 1996).

Entretanto, alguns estudos vêm demonstrando que essas dietas não funcionam para perder peso em longo prazo, podendo trazer várias doenças deletérias, doenças crônicas não transmissíveis (diabetes mellitus, hipertensão arterial sistêmica, obesidade, doenças cardiovasculares), alterações no metabolismo energético e na composição corporal, aumentar a eficiência calórica, gerar o "efeito sanfona" (ciclo de perda-ganho), além de serem consideradas um dos principais fatores desencadeadores dos transtornos alimentares, especialmente os compulsivos (Alvarenga et al., 2016; Souto \& Ferro-Bucher, 2006).

Tendo em vista essas considerações, os estudos sobre o comportamento alimentar vêm crescendo com a proposta de considerar não apenas às questões biológicas da alimentação e nutrição, mas também os aspectos sociais, culturais e 
emocionais, propondo que o indivíduo mantenha uma sintonia com a comida, a mente e o corpo, dando importância ao prazer e ao equilíbrio do que se come (Alvarenga et al., 2016; Tribole \& Resch, 2012).

Dentre as estratégias utilizadas na área do comportamento alimentar para Promoção à saúde e Prevenção de doenças, destaca-se o Mindful eating, que consiste na habilidade da atenção plena ao comer, sem julgamento ou crítica, considerando as sensações físicas e emocionais sentidas (Framson et al., 2009).

Essa ferramenta utiliza técnicas como a meditação mindfulness, para exercitar a atenção plena à quantidade de comida ingerida, à frequência, os sabores, aos horários e ao modo como as pessoas se sentem após comerem, para que os indivíduos possam usar a comida como satisfação de suas necessidades fisiológicas, obtendo bons resultados para o tratamento dos transtornos compulsivos relacionados à alimentação (Germer, Siegel \& Fulton, 2016; Kabat-Zinn, 1990).

Alguns estudos mostram que a utilização do mindful eating nas doenças crônicas não transmissíveis (obesidade, HAS e DM), possuem resultados positivos na redução no ganho de peso, na fome e na alimentação emocional, proporcionando uma melhor qualidade de vida, além de ser uma intervenção preventiva ou adjuvante no diabetes de tipo I e II e na hipertensão (Haenen, Nyklíček, Van, Pop \& Pouwer, 2016; Mantzios \& Wilson, 2015).

Sendo assim, achou-se pertinente realizar uma revisão integrativa da literatura sobre os principais protocolos de Mindful eating utilizados como ferramenta de mudança de comportamento alimentar e suas repercussões clínicas em populações com excesso de peso, bem como as suas características e especificidades.

\section{Metodologia}

O presente trabalho é uma revisão integrativa, que consiste na busca de artigos com informações mais amplas sobre um determinado assunto de forma sistemática, ordenada e abrangente (Mendes, Silveira \& Galvão, 2008). Caracteriza-se por ser uma pesquisa qualitativa em que compara as ferramentas do mindful eating sobre os indivíduos com excesso de peso, nos quais a interpretação por parte do pesquisador é importante sobre o fenômeno em estudo (Pereira, Shitsuka, Parreira \& Shitsuka, 2018).

De acordo com Souza, Silva e Carvalho (2010) a revisão integrativa é um método que constitui basicamente um instrumento da Prática Baseada em Evidências (PBE). A PBE caracteriza-se por uma abordagem voltada ao cuidado clínico e ao ensino fundamentado no conhecimento e na qualidade da evidência. A metodologia envolve a definição do problema clínico, a identificação das informações necessárias, a condução da busca de estudos na literatura e sua avaliação crítica, a identificação da aplicabilidade dos dados oriundos das publicações e a determinação de sua utilização para o paciente.

Para este estudo foi utilizado as seguintes questões norteadoras:

1- Quais os protocolos de mindful eating vêm sendo mais utilizados na literatura para indivíduos com excesso de peso?

2- Quais as repercussões do uso desses protocolos no comportamento alimentar desses indivíduos?

\subsection{Levantamento de dados}

A pesquisa foi realizada nas principais bases de dados da área da Ciência da Saúde, com o intuito de investigar a utilização de protocolos de Mindful eating no tratamento do excesso de peso. Foram utilizadas as bases de dados: PubMed, ScienceDirect, TripDatabase e Scielo. Os descritores utilizados foram: mindfulness, mindful eating, protocolos, obesidade, excesso de peso, perda de peso. 


\subsection{Seleção dos Estudos}

Foram considerados artigos elegíveis para esta revisão integrativa, estudos originais realizados em humanos adultos e idosos de ambos os sexos, que apresentavam excesso de peso ou obesidade, publicados no período de 2009 a 2019 , que correlacionassem o uso de protocolos de mindful eating com a mudança de comportamento alimentar ou avaliassem as repercussões dos mesmos sobre a composição corporal.

Critérios inelegíveis: 1) artigos não originais; 2) artigos que não contemplasse o período; 3) estudos que utilizassem animais; 4) trabalhos que envolviam crianças e/ou adolescentes; 5) pesquisas que não envolvessem os protocolos de mindful eating.

\section{Resultados}

Foram identificados inicialmente 2583 artigos nas bases de dados pesquisadas. Sendo 512 na Pubmed, 42 na Scielo, 1501 no ScienceDirect e 528 no TripDatabase. Após a leitura de títulos e resumos, remoção de duplicatas e com acesso restrito, foram selecionados 30 artigos. Posteriormente, foram excluídos 22 artigos que não abordavam os critérios de elegibilidade estabelecidos. Assim, 8 artigos foram selecionados por preencherem os critérios de inclusão para a produção da revisão (Figura 1).

A análise permitiu a caracterização dos estudos sobre a utilização dos protocolos de mindful eating de acordo com título, o autor, o ano, o protocolo e tempo de intervenção, as (s) doenças (s) analisadas e os principais resultados encontrados. Todas as pesquisas analisadas foram realizadas em países de língua estrangeira (EUA e Canadá), em populações com obesidade, durante o período de 2012 a 2017 (Quadro 1).

De modo a ilustrar o processo de seleção dos estudos, foi utilizado um diagrama de fluxo para identificação do estudo - adaptado por Moher, Liberati, Tetzlaff e Altman (2009) (Figura 1).

Figura1. Fluxo para identificação e seleção dos estudos ( $\mathrm{n}$ = número de artigos).

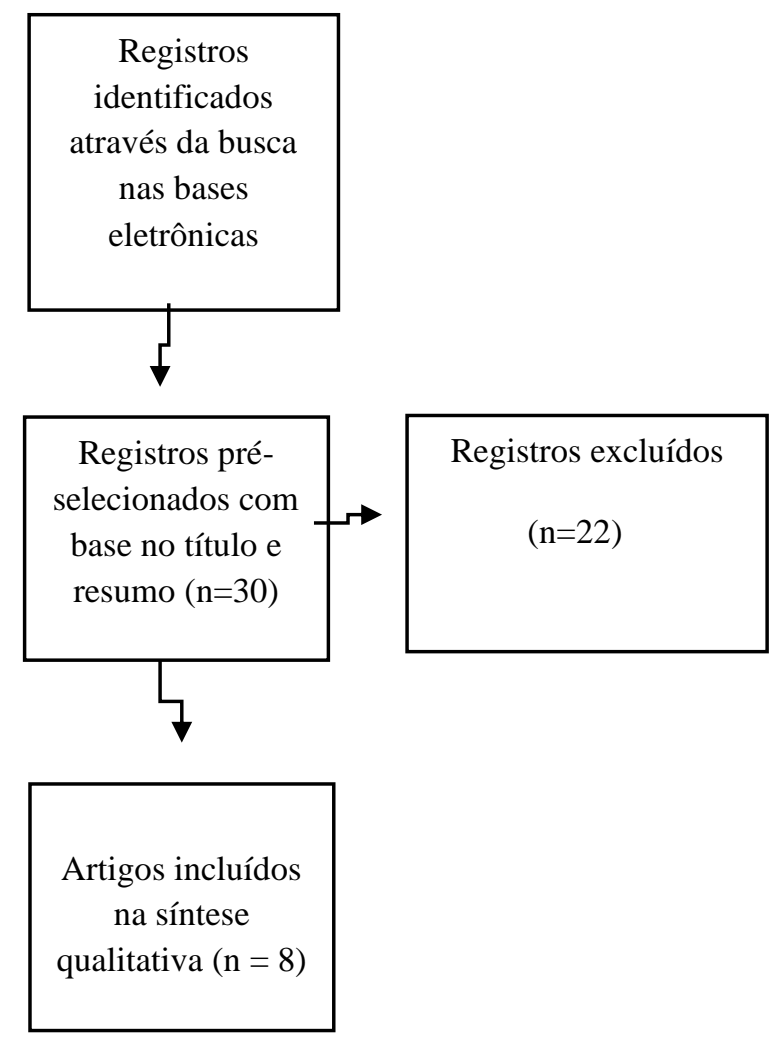

Fonte: Adaptado por Moher et al. (2009). 


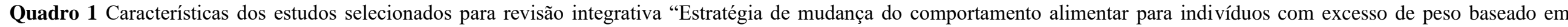
Mindful Eating: Uma revisão integrativa da literatura”.

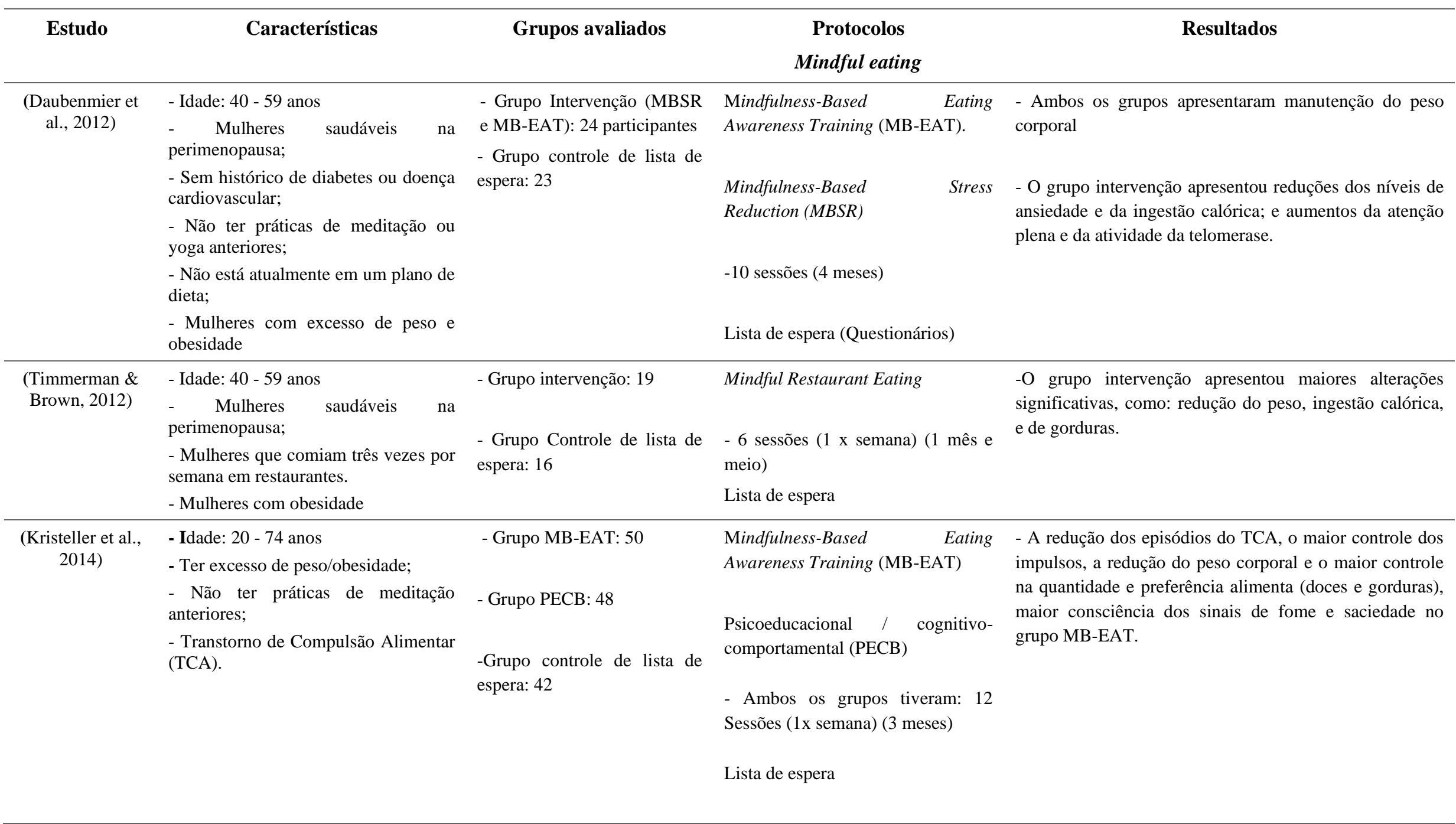




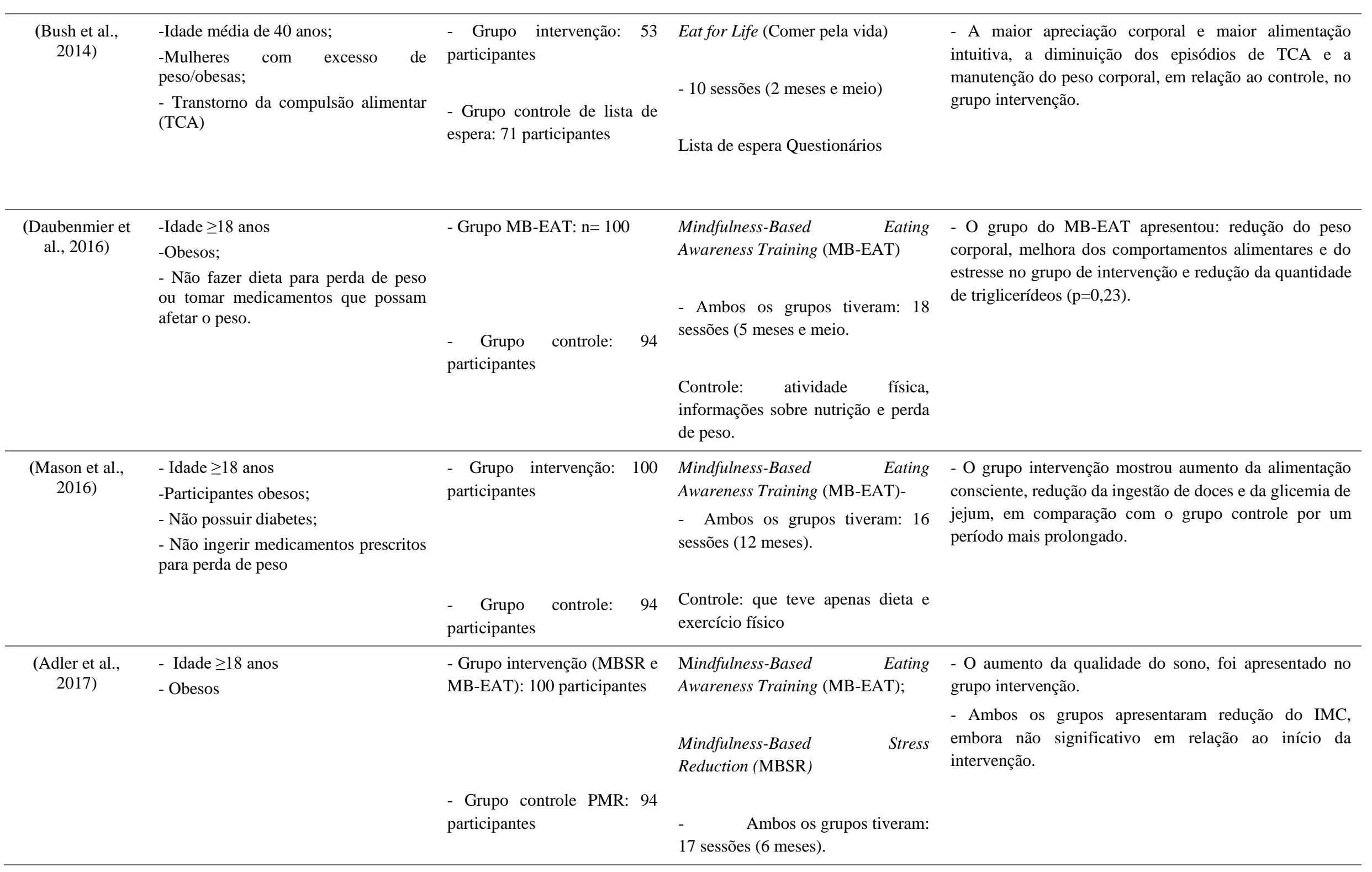


Research, Society and Development, v. 10, n. 3, e36110313465, 2021

(CC BY 4.0) | ISSN 2525-3409 | DOI: http://dx.doi.org/10.33448/rsd-v10i3.13465

\begin{tabular}{|c|c|c|c|c|}
\hline & & & $\begin{array}{l}\text { Controle: } \quad \text { treinamento } \quad \mathrm{em} \\
\text { relaxamento muscular progressivo } \\
(\mathrm{PMR})\end{array}$ & \\
\hline \multirow{5}{*}{$\begin{array}{l}\text { (Wnuk et al., } \\
\text { 2017) }\end{array}$} & -Idade $\geq 18$ anos & \multirow{5}{*}{$\begin{array}{l}\text { - Grupo intervenção: } 28 \\
\text { participantes. }\end{array}$} & Mindfulness-Based Eating & - Melhora significativa da compulsão alimentar e da \\
\hline & - Participantes obesos & & Awareness Training (MB-EAT) & \\
\hline & - 1 Ano ou mais de cirurgia bariátrica & & & - Manutenção do peso. \\
\hline & & & \multirow[t]{2}{*}{ - 8 sessões (4 meses) } & -Melhora no comportamento alimentar. \\
\hline & $\begin{array}{l}\text {-Dificuldades } \\
\text { autorreferidas }\end{array}$ & & & \\
\hline
\end{tabular}

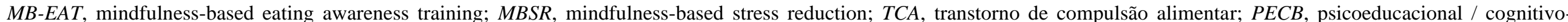
comportamental; $P M R$, treinamento em relaxamento muscular progressivo; n, número de participantes por grupo.

Fonte: Bezerra, M. B. G. S. B. 
As descrições dos trabalhos analisados serão apresentadas abaixo de acordo com cada protocolo e em ordem cronológica:

Os protocolos de Mindful eating existentes atualmente com embasamento científico são (Figura 2): MindfulnessBased Eating Awareness Training (Treinamento de Consciência Alimentar Baseada em Mindfulness, MB-EAT); Mindful Eating-Conscious Living (Vida Consciente da Alimentação Consciente, ME-CL); Mindful Restaurant Eating e Eat For Life (Comer para vida).

Figura 2. Diagrama dos protocolos de Mindful eating utilizados para indivíduos com excesso de peso e suas particularidades.

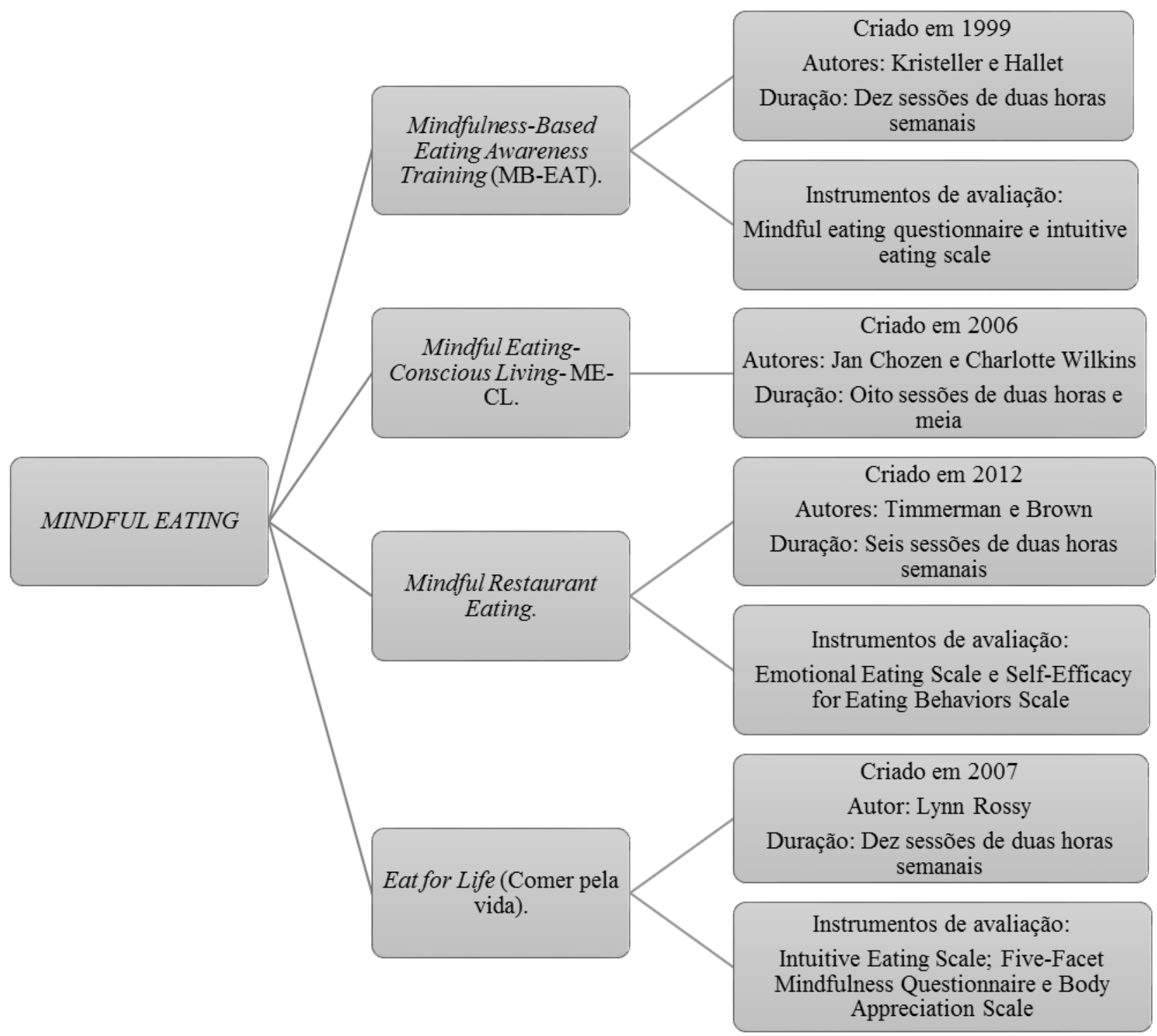

Fonte: Bezerra, M. B. G. S. B.

\subsection{Estudos que utilizaram o Protocolo Mindfulness-Based Eating Awareness Training (MB-EAT)}

O Mindfulness-Based Eating Awareness Training (MB-EAT), objetiva uma alimentação consciente, através de escolhas e consumo alimentares, com a atenção nos sinais de fome e saciedade, estando ciente das sensações fisiológicas e 
emocionais decorrentes da ingestão alimentar (Hendrickson \& Rasmussen, 2013; Miller, Kristeller, Headings \& Nagaraja, 2014) (Figura 2). Foi encontrado na literatura 6 trabalhos que utilizavam o MB-EAT, durante o período de 2012 a 2017.

Daubenmier et al., no ano de 2012, realizou um estudo piloto randomizado controlado para analisar os efeitos de uma intervenção que integrava componentes dos programas de Redução do Estresse Baseada na Atenção Plena (MBSR) e do Treinamento de Conscientização Alimentar Baseada na Atenção Plena (MB-EAT), na atividade da telomerase (preserva o comprimento dos telômeros, a função celular saudável e a função imune a longo prazo; e seu estado de atividade, mostra o estilo de vida e fatores metabólicos que afetam o envelhecimento celular) nas mulheres com excesso de peso e obesidade (Blackburn, 2000; Daubenmier et al., 2012).

As intervenções ocorreram durante quatro meses, consistindo em nove aulas de 2,5 horas e um dia silencioso de 7 horas de pratica de meditação guiada. Foram recrutados 47 participantes do sexo feminino, os quais foram divididos em dois grupos, um grupo de intervenção $(n=24)$ e um grupo controle $(n=23)$. O grupo de intervenção participou de meditações sentados, escaneamento corporal, práticas alimentares conscientes, que incluíam prestar atenção nas sensações físicas de fome e saciedade. Já o controle foi designado para a lista de espera, recebendo uma versão da intervenção mindfulness após a conclusão de todas as avaliações pós-intervenção (Daubenmier et al., 2012).

Não foi apresentada diferença na composição étnica entre os grupos, se identificaram como brancos, e possuíam IMC médio de $31,2 \pm 4,8 \mathrm{~kg} / \mathrm{m}^{2}$. Os resultados demonstraram que o grupo de intervenção mostrou maior redução nos níveis de ansiedade, maior aumento na atenção plena e maior atividade da telomerase do pré ao pós-intervenção, em comparação com o grupo controle. Ambos os grupos mantiveram o peso durante o estudo e apresentaram redução da ingestão calórica, cerca de $293 \pm 442$ calorias (Daubenmier et al., 2012).

No ano de 2014, Kristeller, Wolever e Sheets, realizaram um estudo randomizado com indivíduos obesos e com transtornos de compulsão alimentar periódica (TCAP), com idades entre 20 a 74 anos, com o objetivo de comparar a eficácia do Mindfulness-Based Eating Awareness Training (MB-EAT), em comparação com uma intervenção psicoeducacional / cognitivo-comportamental (PECB) e um controle da lista de espera. Foram recrutados 140 participantes, que foram chamados uma semana antes para o preenchimento dos questionários de alimentação consciente e escala de alimentação intuitiva. Eles foram divididos em três grupos: MB-EAT $(n=50)$, envolvendo meditações de atenção plena e guiadas, mini meditações, exercícios de conscientização concentrando em sinais físicos de fome e saciedade; o PECB (n=48), utilizando exercícios de controle de porções, condicionamento físico, gerenciamento de estresse e recomendações psicossociais para gerenciar a compulsão alimentar; e controle da lista de espera $(n=42)$, não receberam tratamento durante o período, só recebendo acesso à alimentação consciente ou ao treinamento do PECB posteriormente (Kristeller et al., 2014).

Ambos os grupos participaram da intervenção durante 12 semanas com duração de $2 \mathrm{~h}$, incentivados a não focarem sua atenção na perda de peso, concentrando-se em se tornarem mais conscientes dos padrões de alimentações inadequadas e adquirirem mudanças mais sustentáveis (Kristeller et al., 2014).

A intervenção demonstrou que o grupo do MB-EAT teve uma melhora no controle dos impulsos, na redução nos autojulgamentos negativos, na capacidade de identificar e usar a consciência sobre os sinais de fome e saciedade, na redução dos comportamentos do transtorno de compulsão alimentar periódica, em comparação com os grupos PECB e controle. Também foi demonstraram que apesar de não ter ocorrido diferenças significativas, os integrantes do MB-EAT tiveram uma maior perda de peso corporal no valor de 5 libras ou mais ( $p<0,05$ ), comparado aos outros grupos (Kristeller et al., 2014).

Além disso, os participantes do MB-EAT relataram um maior controle no consumo de doces e alimentos com alto teor de gordura, tanto em relação a quantidade quanto na preferência, relatando que porções menores já os satisfaziam (Kristeller et al., 2014). 
No ano de 2016, Daubenmier et al., realizaram um estudo controlado e randomizado com participantes obesos, com idade de 18 anos ou mais, com o objetivo de analisar se a intervenção de Mindfulness-Based Eating Awareness Training (MBEAT), mostrariam maiores melhorias na manutenção da perda de peso. A intervenção ocorreu em 18 sessões com duração de 2 a 2,5 h, por um período de cinco meses e meio, com ou sem componentes de atenção plena. Foram recrutados 194 participantes, os quais foram divididos em dois grupos: MB-EAT $(n=100)$, que incluiu práticas de meditações de controle de estresse e alimentação consciente, mini meditações, caminhadas conscientes que incluía consciência da experiência sensorial, com o intuito de aumentar a conscientização e a autorregulação da fome física e controle ( $n=94)$, que receberam informações sobre nutrição e atividade física, discussão de questões sociais sobre perda de peso, lanches e atividades domésticas, relaxamento muscular progressivo e treinamento de força com faixas de exercícios (Daubenmier et al., 2016).

A idade média dos integrantes foi de 47,2 (13\%), mais da metade era do sexo feminino $(82,5 \%)$, e a média do índice de massa corporal inicial (IMC) foi de 35,7 $\pm 3,5$. O resultado desse estudo mostrou que o grupo de atenção plena teve uma perda de peso de $1,7 \mathrm{~kg}(\mathrm{p}=0,27)$ maior que o grupo controle. Mostrou também que a quantidade de triglicerídeos tiveram uma redução de $9,7 \mathrm{mg} / \mathrm{dl}(\mathrm{p}=0,23)$ no grupo de atenção plena, devido a melhora dos comportamentos alimentares e do estresse no grupo de intervenção (Daubenmier et al., 2016).

No ano de 2016, Mason et al., realizaram um estudo controlado e randomizado para avaliar o efeito de uma intervenção de atenção plena, no consumo de doces e nos níveis de glicose em jejum, em participantes obesos, através do Treinamento de Conscientização Alimentar Baseada na Atenção Plena (MB-EAT). A intervenção ocorreu por um período de 6 meses, com 16 sessões, durante 2 horas (Mason et al., 2016).

Nesse período, foram recrutados 194 participantes, em sua maioria do sexo feminino e brancos, com idade média foi de 47,0 anos e IMC médio inicial de 35,5 $\pm 3,6 \mathrm{~kg} / \mathrm{m}^{2}$, os quais foram divididos em dois grupos: intervenção ( $\mathrm{n}=100$ ), que envolveu intervenções com técnicas da alimentação consciente, meditações alimentares guiadas e de varredura corporal, mini meditações antes das refeições para conscientizar as sensações de fome e saciedade; e controle ativo ( $\mathrm{n}=94)$, envolvendo somente dieta e exercício, recebendo informações adicionais sobre nutrição e atividade física, discussões sobre a escolha de alimentos. Os resultados mostraram que o grupo intervenção teve um maior aumento na alimentação consciente, em comparação com o grupo controle. Ambos os grupos tiveram uma redução na ingestão de doces e da glicemia em jejum, porém o grupo de intervenção manteve essa redução durante os 6 a 12 meses após a intervenção, já o controle nesse período teve um aumento (Mason et al., 2016).

Adler et al. (2017), em pesquisa de caráter controlado e randomizado com o objetivo de analisar os efeitos da intervenção de Treinamento de Conscientização Alimentar Baseada na Atenção Plena (MB-EAT) e Redução do Estresse Baseada na Atenção Plena (MBSR) na qualidade do sono, em pacientes obesos, utilizaram intervenções no período de 6 meses, com 17 sessões, durante 2 horas. Foram avaliados 194 indivíduos, os quais foram divididos em dois grupos: atenção plena ( $\mathrm{n}=100)$, que envolveu práticas do MBSR e MB-EAT, utilizando a meditação da bondade amorosa guiadas, ioga suave, caminhadas conscientes, práticas de atenção plena durante 30 minutos, alimentação consciente e breves mini meditações; e grupo controle ( $\mathrm{n}=94$ ), que contou um treinamento em relaxamento muscular progressivo (PMR), embora em dose mais baixa do que na intervenção de atenção plena e informações nutricionais e de atividade física (Adler et al., 2017).

De acordo com os autores, ambos os grupos não diferiram significativamente nas características sociodemográficas ou clínicas na linha de base. A maioria dos integrantes eram do sexo feminino (80\%), se autorreferiram brancas (59\%), com IMC médio de 35,5 $\pm 3,6 \mathrm{~kg} / \mathrm{m}^{2}$. Os resultados encontrados mostraram um aumento na qualidade do sono no grupo de intervenção, em comparação com o controle, e ambos os grupos houve redução pouco significativa do IMC de $0,23 \mathrm{~kg} / \mathrm{m}^{2},(\mathrm{p}=0,43) \mathrm{em}$ relação ao início da intervenção (Adler et al., 2017). 
Wnuk et al. (2017), realizaram um estudo randomizado com o objetivo de analisar a eficácia do Treinamento de Conscientização Alimentar Baseada na Atenção Plena (MB-EAT) para pacientes em cirurgia pós-bariátrica. A intervenção ocorreu por um período de 4 meses, com 8 sessões, durante 2 horas; O programa constou de componentes experimentais e didáticos, com práticas de atenção plena como meditação da respiração, varredura corporal, meditação do perdão, assim como práticas alimentares conscientes com variados tipos de alimentos, com restrições alimentares para os participantes pósoperatórios. Participaram 28 pessoas, com idade média de 55,41 anos, do sexo feminino, com IMC médio de 32,82 $\pm 5,31 \mathrm{~kg} /$ $\mathrm{m}^{2}$ e mais da metade se autorreferiam como caucasianos (95\%). Foi demonstrado que houve melhora significativa na compulsão alimentar e na depressão, e houve uma redução na alimentação emocional em resposta a raiva, depressão e ansiedade, mostrando melhora no comportamento alimentar. Porém, não houve alteração do peso corporal (Wnuk et al., 2017).

\subsection{Estudos que utilizaram o Protocolo Mindful Eating-Conscious Living (ME-CL)}

O Mindful Eating-Conscious Living (ME-CL) foi desenvolvido com o objetivo de ensinar as habilidades que levam ao aumento da consciência do comer, das emoções e do autojulgamento negativo, para que assim haja uma mudança de comportamentos e reduzir a alimentação automática, permitindo que os participantes identifiquem os sinais de fome e saciedade (Dalen et al., 2010; Smith, Shelley, Leahigh \& Vanleit, 2006) (Figura 2).

Não foram encontrados artigos publicados com este Protocolo, provavelmente devido ao seu caráter atual e as pesquisas utilizando o mesmo, ainda estarem em andamento (informação dos autores- Bays e Char), sendo necessário um maior investimento nessa área de estudo.

\subsection{Estudos que utilizaram o Protocolo Mindful Restaurant Eating}

O Mindful Restaurant Eating tem como objetivo desenvolver habilidades necessárias para reduzir a ingestão calórica e de gordura ao comer em restaurantes, prevenindo o ganho de peso, mantendo o prazer em comer fora. Esse protocolo utiliza como base: conhecimento do teor calórico e de gordura dos alimentos; estratégias de mudança de comportamentos; atividades de acordo com as preferências pessoais; e meditação alimentar consciente (Glanz, Basil, Maibach, Goldberg \& Snyder, 1998) (Figura 2). Durante a busca na literatura foi encontrado um trabalho que utilizou esse protocolo, no ano de 2012, que será descrito posteriormente.

No ano de 2012, Gayle Timmerman e Adama Brown, realizaram um estudo de controle randomizado com mulheres saudáveis na perimenopausa que comiam fora pelo menos três vezes por semana, com idades entre 40 e 59 anos, usando o Mindful Restaurant Eating.

Foram recrutados 35 participantes, do sexo feminino, saudáveis, com idade média da amostra participante foi de 49,6 anos. Foram divididos em dois grupos: intervenção $(n=19)$, que participaram das 6 sessões de meditações, e o controle da lista de espera $(n=16)$, que foram recrutados na sexta semana após a última sessão. A intervenção contou com sessões de meditação guiadas presenciais e em casa e discussões sobre os princípios do controle de peso ao comer fora, durante 6 sessões semanais de duração de $2 \mathrm{~h}$ (Timmerman \& Brown, 2012).

Os resultados mostraram que o grupo de intervenção apresentou redução de peso significativamente de $1,7 \mathrm{~kg}$ ( $\mathrm{p}=0,03$ ), redução da ingestão diária de calorias (menos 346,84 calorias) e de gorduras (menos 18,27 gramas) maiores em comparação com o grupo controle. Além disso, aumentou a autoeficácia ao perceber a dieta, e houve redução das barreiras que impediam o controle do peso, sendo mais perceptíveis a causa de uma ingestão descontrolada (Timmerman \& Brown, 2012). 


\subsection{Estudos que utilizaram o Protocolo Eat for Life}

O Eat for Life (Comer pela vida) tem como objetivo ensinar as pessoas a comer de forma consciente e intuitiva, amar seus corpos e encontrar um significado mais profundo em suas vidas (Bush, Rossy, Mintz \& Schopp, 2014). Ele conta com princípios da alimentação intuitiva e da atenção plena, que defendem uma alimentação baseado nos sinais de fome e saciedade, atenção no momento presente e sem julgamentos, exposição de pensamentos e emoções, flexibilidade cognitiva e comportamental (Shapiro, Carlson, Astin \& Freedman, 2006; Tribole \& Resch, 1995). Durante a busca na literatura foi encontrado um trabalho que utilizou esse protocolo, no ano de 2014, que será descrito posteriormente.

No ano de 2014, Bush et al., realizaram um estudo com mulheres com excesso de peso/obesas, com idade média de 40 anos, com transtorno da compulsão alimentar periódica (TCAP), usando o Eat for Life. O objetivo do estudo foi analisar se o treinamento em alimentação intuitiva e atenção plena aprimorariam a apreciação do corpo e no comportamento alimentar (Bush et al., 2014).

Foram recrutados 124 participantes, que foram divididos em gois grupo: intervenção $(n=53)$, que preencheram questionários pré-intervenção (Intuitive Eating Scale, Five-Facet Mindfulness Questionnaire e Body Appreciation Scale), que foi seguida de sessões de meditações guiadas feitas em grupos e recomendadas a prática em casa, discussões sobre o princípio da alimentação intuitiva, leituras do manual do Eat for Life, e pós-questionários; e controle ( $\mathrm{n}=71$ ), receberam os préquestionários e pós-questionários com 10 semanas de intervalo (Bush et al., 2014).

Após o período da intervenção o grupo intervenção obtiveram níveis mais elevados de apreciação corporal e alimentação intuitiva, e uma diminuição dos transtornos de compulsão alimentar, em comparação com o controle. Eles também passaram a ter mais conhecimento sobre quando e quanto comer, prestando atenção nas suas sensações de fome e saciedade, beneficiando sua autorregulação na hora da alimentação, resultando na manutenção do peso corporal, comparado ao outro grupo (Bush et al., 2014).

\section{Discussão}

No presente estudo, foi analisada a eficácia dos protocolos de mindful eating no tratamento de indivíduos com excesso de peso, verificando os seus possíveis benefícios sobre o comportamento alimentar.

As pesquisas, nos diversos artigos apresentados, demonstraram, em sua maioria, significativas mudanças no comportamento alimentar, na atenção plena, diminuição dos episódios de compulsão alimentar. Entretanto, em relação ao peso corporal, os resultados são diferentes e às vezes conflitantes, que vão de acordo com as autoras Kristeller e Wolever (2011), quando afirmam que as práticas de alimentação consciente são um caminho para o aumento da consciência das sensações de fome e saciedade, o que ajuda aos indivíduos a equilibrar suas respostas alimentares.

Como Kristeller e Hallett (1999) sugerem, uma intervenção alimentar tendo como base a atenção plena, pode trazer alterações importantes, rápidas e contínuas na compulsão alimentar e características que envolvem o comportamento alimentar. As práticas de alimentação consciente permitem que os indivíduos consigam entender quando e quanto comer durante as refeições, permitindo que restaurem o equilíbrio entre os sinais físicos internos (fome e saciedade) e as sensações emocionais, sendo essencial para o comportamento alimentar, ajudando em maior controle do peso (Kristeller \& Hallett, 1999).

Estudos mostraram que as intervenções com atenção plena ajudam a maiores percepções de pensamentos, emoções e sensações angustiantes, responsáveis pelo alto consumo de alimentos, proporcionando um maior controle da angústia e interrupção do aumento da alimentação em resposta a sinais não físicos (Ouwens, Schiffer, Visser, Raeijmaekers \& Nyklíčk, 2015).

Uma revisão realizada por Warren, Smith e Ashwell (2017), com trabalhos que utilizaram intervenções de atenção plena em participantes com sobrepeso ou obesidade, mostrou que esses programas podem ser eficazes para redução de 
compulsões alimentares, maior apreciação corporal, maior controle de ingestão alimentar. Porém, alguns também mostraram que um maior nível de alimentação intuitiva, poderia estar associado ao aumento do consumo de alimentos em um ambiente permissivo. Além de mostrar que as intervenções não são determinantes para uma perda de peso significativa, podendo haver só a sua manutenção (Warren, Smith \& Ashwell, 2017).

Pesquisas realizadas com pacientes bariátricos, mostraram que o aumento da consciência e da atenção no momento da alimentação, promove menos compulsões e alimentações emocionais, além de gerar bem-estar mental e físico (Khan \& Zadeh, 2014; Levin, Dalrymple, Himes \& Zimmerman, 2014).

Outros estudos que avaliaram o uso das intervenções de mindful eating, mostraram resultados positivos nas mudanças da autoeficácia para perda de peso, no aumento da atenção plena, na alimentação consciente, no comportamento alimentar, através de uma maior conscientização e atenção ao comer. Utilizado, para isso, práticas de meditação guiada, alimentação consciente, prestando atenção nas sensações físicas de fome e saciedade. Comprovando ser uma alternativa para substituir as dietas restritivas, que alteram a quantidade ou a qualidade dos alimentos ingeridos, restringindo o consumo de determinados alimentos, para controlar ou alterar o peso corporal, podendo a longo prazo trazer problemas como o transtorno de compulsão alimentar - TCAP (Bays, 2009; Kidd, Graor \& Murrock, 2013).

Vale salientar as possíveis modulações neuroendócrinas e de neuroplasticidade que devem ocorrer nos indivíduos submetidos às intervenções de mindful eating, como: maior ativação das áreas do hipocampo, insula e córtex cingulado anterior, permitindo um maior controle das emoções frente a determinados estímulos; menor ativação da amigdala, que promove uma melhora do comportamento, permitindo que a alimentação não seja realizada emocionalmente, uma vez que com sua menor ativação há uma regulação das emoções. Estas modulações provavelmente devem estar envolvidas com as respostas na mudança do comportamento alimentar os estudos analisados, entretanto estes parâmetros (neuromodulação e neuroendócrinas) não foram avaliadas nestes estudos (Decety \& Jackson, 2004; Hölzel et al., 2011).

Os protocolos de mindful eating consistem em alternativas atuais para melhorar a aceitação do "eu", permitindo uma maior capacidade de fazer escolhas mais saudáveis, contribuindo para a mudança de comportamento alimentar. Sugerindo que a inclusão de componentes alimentares conscientes nos programas de redução de peso, possam melhorar o comportamento alimentar e o controle do peso corporal (Alberts, Thewissen \& Raes, 2012).

Pesquisas utilizando protocolos de mindful eating ainda são recentes, apresentando poucos trabalhos publicados na literatura, especialmente nacional, o que torna um fator limitante para a compreensão e comparação dos resultados.

No presente estudo foi possível identificar que o protocolo mais utilizado para o tratamento de indivíduos com excesso de peso foi o Mindfulness-Based Eating Awareness Training (MB-EAT), apresentando maiores quantidades de trabalhos nas bases de dados pesquisadas.

Ressalta-se ainda, a necessidade de estudos com amostras mais representativas, além da necessidade de pesquisas com desenhos metodológicos controlados e padronizados (estudos randomizados e controlados), bem como da análise de outras variáveis envolvidas no comportamento alimentar e na fisiologia dos processos metabólicos, para que seja possível fazer as devidas comparações e conclusões sobre os benefícios das intervenções baseadas nos protocolos de Mindful eating na população adulta com excesso de peso.

\section{Conclusão}

A análise do presente estudo, demonstrou a eficácia da utilização dos protocolos de mindful eating nos indivíduos com excesso de peso como intervenção de promoção à saúde, apresentando melhorias na manutenção ou redução do peso corporal, no comportamento alimentar, na autoaceitação corporal, bem como na diminuição dos episódios compulsivos, o que 
parece ser uma boa estratégia para ser utilizada nessa população em vários contextos, especialmente nas políticas públicas de saúde que visam o enfrentamento à obesidade e suas comorbidades.

Entre os protocolos validados, o que apresenta maior número de publicações na literatura científica é o MindfulnessBased Eating Awareness Training (MB-EAT). Dessa forma, percebe-se a necessidade de mais estudos que abordem os demais protocolos existentes, para permitir a análise dos seus efeitos e fortalecer a sua eficácia como estratégia de mudança de comportamento alimentar e suas interfaces.

Sugere-se também que, embora o mindful eating promova a melhora da relação dos indivíduos com a alimentação e com o seu corpo, são necessários investimentos em estudos clínicos com adequados controle metodológico, para que possam ratificar a sua utilização como uma estratégia para melhora da qualidade de vida das pessoas com excesso de peso, em vários cenários e contextos (diferentes populações e ciclos de vida, etnias e situações clínicas).

\section{Referências}

Adler, E., Dhruva, A., Moran, P., Daubenmier, J., Acree, M., Epel, E. S. \& Hecht, F. M. (2017). Impact of a Mindfulness-Based Weight-Loss Intervention on Sleep Quality Among Adults with Obesity: Data from the SHINE Randomized Controlled Trial. J Altern Complement Med., 3 (3), 188-195. https://doi.org/10.1089/acm.2016.0141.

Alberts, H. J. E. M., Thewissen, R., \& Raes, L. (2012). Dealing with problematic eating behavior: The effects of a mindfulness-based intervention on eating behavior, food cravings, dichotomous thinking and body image concern. Appetite, 58, 847-851. https://doi.org/10.1016/j.appet.2012.01.009.

Alvarenga, M., Polacow, V., \& Scagliusi, F. (2016). Dieta e seus efeitos no comportamento alimentar. Manole Ltda.

Bays, J. C. (2009). Mindful eating - a guide to discovering a healthy and joyful relationship with food. Shambhala.

Blackburn, E. H. (2000). Estados dos telômeros e destino das células. Natureza, 408 (6808), 53-56. https://doi.org/10.1038/35040500.

Bush, H., Rossy, L., Mintz, L. B., \& Schopp, L. (2014). Eat for Life: A Work Site Feasibility Study of a Novel Mindfulness-Based Intuitive Eating Intervention. Am J Health Promot, 28 (6). https://doi.org/10.4278/ajhp.120404-QUAN-186.

Dalen, J., Smith, B. W., Shelley, B. M., Sloan, A. L., Leahigh, L., \& Begay, D. (2010). Pilot study: Mindful Eating and Living (MEAL): Weight, eating behavior, and psychological outcomes associated with a mindfulness-based intervention for people with obesity. Complement Ther Med., 18 (6), 260-264. https://doi.org/10.1016/j.ctim.2010.09.008.

Daubenmier, J., Lin, J., Blackbum, E., Hecht, F. M., Kristeller, J., Maninger, N. Epel, E. (2012). Changes in stress, eating, and metabolic factors are related to changes in telomerase activity in a randomized mindfulness intervention pilot study. Psychoneuroendocrinology, 37 (7), 917-928. https://doi.org/10.1016/j.psyneuen.2011.10.008.

Daubenmier, J., Moran, P. J., Kristeller, J., Acree, M., Bacchetti, P., Kemeny, M. E., Hecht, F. M. (2016). Effects of a mindfulness-based weight loss intervention in adults with obesity: A randomized clinical trial. Obesity (Silver Spring), 24 (4), 794-804. https://doi.org/10.1002/oby.21396.

Decety, J., \& Jackson, P. L. (2004). The functional architecture of human empathy. Behav Cog Neurosci Rev.. 3 (2), 71-100. https://doi.org/10.1177/1534582304267187.

Foxcroft, L. (2013). A tirania das dietas. Três Estrelas.

Framson, C., Kristal, A. R., Schenk, J. M., Littman, A. J., Zeliadt, S., \& Benitez, D. (2009). Development and validation of the mindful eating questionnaire. J Am Diet Assoc., 109, 1439-44. https://doi.org/10.1016/j.jada.2009.05.006.

Germer, C. K., Siegel, R. D., \& Fulton, P. R. (2016). Mindfulness e psicoterapia. Artmed.

Glanz, K., Basil, M., Maibach, E., Goldberg, J., \& Snyder, D. (1998). Why Americans eat what they do: Taste, nutrition, cost, convenience, and weight control concerns as influences on food consumption. J Am Diet Assoc., 98 (10), 1118-26. https://doi.org/10.1016/S0002-8223(98)00260-0.

Haenen, S., Nyklíček, I., Van, S. J., Pop, V., \& Pouwer, F. (2016). Mindfulness facets as differential mediators of short and long-term effects of MindfulnessBased Cognitive Therapy in diabetes outpatients: Findings from the DiaMind randomized trial. J Psychosom Res., (85), 44-50. https://doi.org/10.1016/j.jpsychores.2016.04.006.

Hendrickson, K. L., \& Rasmussen, E. B. (2013). Effects of mindful eating training on delay and probability discounting for food and money in obese and healthy-weight individuals. Behav Res Ther, 51, 399-409. https://doi.org/10.1016/j.brat.2013.04.002.

Hölzel, B. K., Carmody, J., Vangel, M., Congleton, C., Yerramsetti, S. M., Gard, T. \& Lazar S. W. (2011). Mindfulness practice leads to increases in regional brain gray matter density. Psychiatry Res Neur., 191, 36-43. https://doi.org/10.1016/j.pscychresns.2010.08.006.

Kabat-zinn, J. (1990). Full catastrophe living: using the wisdom of your body and mind to facestress, pain, and illness. Delta.

Khan, Z., \& Zadeh, Z. (2014). Mindful eating and its relationship with mental well-being. Social and Behavioral Sciences, 159, 69-73. https://doi.org/10.1016/j.sbspro.2014.12.330. 
Kristeller, J. L., \& Hallett, C. B. (1999). An exploratory study of a meditation-based intervention for binge eating disorder. J Health Psychol., 4, 357-363. https://doi.org/10.1177/135910539900400305.

Kristeller, J. L., \& Wolever, R. Q. (2011). 'Mindfulness-Based Eating Awareness Training for Treating Binge Eating Disorder: The Conceptual Foundation'. Eating Disorders, 19 (1), 49 - 61. https://doi.org/10.1080/10640266.2011.533605.

Kristeller, J., Wolever, R. Q., \& Sheets, V. (2014). Mindfulness-Based Eating Awareness Training (MB-EAT) for Binge Eating: A Randomized Clinical Trial. Mindfulness. Springer Science, 5 (3), 282-297. https://doi.org/10.1007/s12671-012-0179-1.

Kidd, L. I., Graor, C. H., \& Murrock, C. J. (2013). A Mindful Eating Group Intervention for Obese Women: A Mixed Methods Feasibility Study. Arch Psychiatr Nurs., 27, 211-218. https://doi.org/10.1016/j.apnu.2013.05.004.

Levin, M. E., Dalrymple, K., Himes, S., \& Zimmerman, M. (2014). Which facets of mindfulness are related to problematic eating among patients seeking bariatric surgery? Eating Behav., 15, 298-305. https://doi.org/10.1016/j.eatbeh.2014.03.012.

Levy, R. B., Claro, R. M., Mondini, L., Sichieri, R., \& Monteiro, C. A. (2012). Distribuição regional e socioeconômica da disponibilidade de alimentos no Brasil, 2008-2009. Ver. Saúde Pública, 46 (1), 6-15. https://doi.org/10.1590/S0034-89102011005000088.

Mantzios, M., \& Wilson, J. C. (2015). Mindfulness, eating behaviours, and obesity: a review and reflection on current findings. Curr Obes Rep., 4, 141-6. https://doi.org/10.1007/s13679-014-0131-x.

Mason, A. E., Epel, E. S., Kristeller, J., Moran, P. J., Dallman, M., Lustig, R. H. \& Daubenmier, J. (2016). Effects of a mindfulness-based intervention on mindful eating, sweets consumption, and fasting glucose levels in obese adults: data from the SHINE randomized controlled trial. J Behav Med., 39 (2), 20113. https://doi.org/10.1007/s10865-015-9692-8.

Mendes, K. D. S., Silveira, R. C. C. P., \& Galvão, C. M. (2008). Revisão integrativa: método de pesquisa para a incorporação de evidências na saúde e na enfermagem. Texto Contexto Enfermagem, 17 (4), 758-64. https://doi.org/10.1590/S0104-07072008000400018.

Miller, C. K., Kristeller, J. L., Headings, A., \& Nagaraja, H. (2014). Comparison of a mindful eating intervention to a diabetes self-management intervention among adults with type 2 diabetes: a randomized controlled trial. Health Educ Behav., 41, 145-154. https://doi.org/10.1177/1090198113493092.

Moher, D., Liberati, A., Tetzlaff, J., \& Altman, D. G. (2009). Preferred reporting items for systematic reviews and meta-analyses: the PRISMA statement. PLOS Medicine, 6 (7). https://doi.org/10.1371/journal.pmed.1000097.

Ouwens, M. A., Schiffer, A. A., Visser, L. I., Raeijmaekers, N. J. C., \& Nyklíčk, L. (2015). Mindfulness and eating behaviour styles in morbidly obese males and females. Appetite, 87, 62-67. https://doi.org/10.1016/j.appet.2014.11.030.

Pereira, A. S., Shitsuka, D. M., Parreira, F. J., \& Shitsuka, R. (2018). Metodologia da pesquisa científica [Versão digital em ebook]. Recuperado de https://repositorio.ufsm.br/bitstream/handle/1/15824/Lic_Computacao_Metodologia-Pesquisa-Cientifica.pdf.

Pinheiro, A. R. O., Freitas, S. F. T., \& Corso, A. C. T. (2004). Uma abordagem epidemiológica da obesidade. Rev. Nutr. 17 (4), $523-33$. https://doi.org/10.1590/S1415-52732004000400012.

Rozin, P., Ashmore, M., \& Markwith, M. (1996). Lay American conceptions of nutrition: dose insensivity, cathegorical thinking, contagion, and monotonic mind. J of Health Psychology, 15 (6), 438-47. https://doi.org/10.1037/0278-6133.15.6.438.

Shapiro, S. L., Carlson, L. E., Astin, J. A., \& Freedman, B. (2006). Mechanisms of mindfulness. J Clin Psychol., 62, 373-386. https://doi.org/10.1002/jclp.20237.

Smith, B. W., Shelley, B. M., Leahigh, L., \& Vanleit, B. (2006). A preliminary study of the effects of a modified mindfulness intervention on binge eating. Complement Health Practice, 1 (3), 133-43. https://doi.org/10.1177/1533210106297217.

Souto, S., \& Ferro-Bucher, J. S. N.S (2006). Práticas indiscriminadas de dietas de emagrecimento e o desenvolvimento de transtornos alimentares. Rev. Nutr. 19 (6), 693-704. https://doi.org/10.1590/S1415-52732006000600006.

Souza, M. T., Silva, M. D., \& Carvalho, R. (2010). Revisão integrativa: o que é e como fazer. Einstein, 8(1), 102-106. https://doi.org/10.1590/s1679$45082010 \mathrm{rw} 1134$.

Timmerman, G. M., \& Brown, A. (2012). The effect of a mindful restaurant eating intervention on weight management in women. J Nutr Educ Behav., 44 (1), 22-8. https://doi.org/10.1016/j.jneb.2011.03.143.

Tribole, E., \& Resch, E. (1995). Intuitive Eating: A Recovery Book for the Chronic Dieter. St. Martin's Press.

Tribole, E., \& Resch, E. (2012). Intuitive eating - A revolutionary program that works. St. Martin's Griffin.

Warren, J., Smith, N., \& Ashwell, M. (2017). A structured literature review on the role of mindfulness, mindful eating and intuitive eating in changing eating behaviours: Effectiveness and associated potential mechanisms. Nutr Res Rev., 30 (2), 272-283. https://doi.org/10.1017/S0954422417000154.

Wnuk, S. M., Du, C. T., Exan, J. V., Wallwork, A., Tremblay, L., Kowgier, M., \& Sockalingam, S. (2017). Mindfulness-Based Eating and Awareness Training for Post-Bariatric Surgery Patients: a Feasibility Pilot Study. Mindfulness, 9 (3), 949-960. https://doi.org/10.1007/s12671-017-0834-7.

World Health Organization (2010). Creating an enabling environment for population-based salt reduction strategies: report of a joint technical meeting held by WHO and the Food Standards Agency, United Kingdom. Geneva: WHO. https://apps.who.int/iris/handle/10665/44474. 